\title{
Preoperative left ventricular energy loss in the operating theater reflects subjective symptoms in chronic aortic regurgitation
}

\author{
Atsushi Kainuma ${ }^{1}$, Keiichi Itatani ${ }^{1}$, Koichi Akiyama ${ }^{1}$, Yoshifumi Naito ${ }^{1}$, Maki Ishii ${ }^{1}$, \\ Masaru Shimizu ${ }^{1}$, Junya Ohara ${ }^{1}$, Naotoshi Nakamura ${ }^{2}$, Yasufumi Nakajima ${ }^{3}$, Satoshi \\ Numata $^{1}$, Hitoshi Yaku ${ }^{1}$, and Teiji Sawa ${ }^{1}$ \\ ${ }^{1}$ Kyoto Prefectural University of Medicine \\ ${ }^{2}$ Osaka University \\ ${ }^{3}$ Kansai Medical University
}

August 23, 2020

\begin{abstract}
Objective: There is currently no subjective, definitive evaluation method for therapeutic indication other than symptoms in aortic regurgitation. Energy loss, a novel parameter of cardiac workload, can be visualized and quantified using echocardiography vector flow mapping. The purpose of the present study was to evaluate whether energy loss in patients with chronic aortic regurgitation can quantify their subjective symptoms more clearly than other conventional metrics. Methods: We studied 15 patients undergoing elective aortic valve surgery for aortic regurgitation. We divided the patients into symptomatic and asymptomatic groups using their admission records. We analyzed the mean energy loss in one cardiac cycle using transesophageal echocardiography during the preoperative period. The relationships between symptoms, energy loss, and other conventional metrics were statistically analyzed. Results: There were seven and eight patients in the symptomatic and asymptomatic groups, respectively. The mean energy loss of one cardiac cycle was higher in the symptomatic group (121 [96 to 184]) than in the asymptomatic group (87 [80 to 103]) ( $\mathrm{p}=0.040)$, whereas the diastolic diameter was higher in the asymptomatic group (65 [59 to 78$]$ ) than in the symptomatic group (57 [51 to 57]) ( $\mathrm{p}=0.040)$. There was no significant difference between the symptomatic and asymptomatic groups in terms of other conventional metrics. Conclusions: An energy loss can quantify patients' subjective symptoms more clearly than other conventional metrics. The small sample size is the primary limitation of our study, further studies assessing larger cohort of patients are warranted to validate our findings.
\end{abstract}

\section{Introduction}

There is no subjective, definitive evaluation method for therapeutic indication other than presenting symptoms in aortic regurgitation (AR). According to the 2014 American College of Cardiology/American Heart Association guidelines for the management of chronic AR, the indication for aortic valve surgery is mainly based on the presenting symptoms; the indications in asymptomatic patients are decreased and impaired systolic function or a highly dilated left ventricular chamber ${ }^{1}$. In order not to miss the optimal timing of the surgical indication for improved postoperative patient prognosis, a novel parameter to estimate not the current cardiac function but the workload itself should be required.

Echocardiography vector flow mapping (VFM) is one of the blood flow visualization techniques which enables evaluation of cardiac energy loss (EL); these can detect the progression of cardiovascular disease ${ }^{2-4}$. Normal pattern vortex and EL reference value of the left ventricle in adults and pediatric patients were confirmed by using $\mathrm{VFM}^{5,6}$. Several studies attempted to elucidate the mechanism of thrombus formation in the left ventricle $^{7,8}$. VFM analysis of valvular ${ }^{9-12}$ and congenital heart disease ${ }^{13-15}$ can reportedly reveal and evaluate 
the effectiveness of the surgical treatment. As it does not require the use of contrast, this VFM technology can be easily used in routine clinical practice to assess ventricular vortices and predict patients' outcome

16 .

Previous studies have already revealed that left ventricular diastolic EL increases proportionally to AR severity owing to turbulent vortex flow ${ }^{17}$. However, there is still limited information about intracardiac flow evaluation in aortic valve disease

16. The aim of the present study was to determine whether left ventricular EL in chronic AR can be an alternative and superior parameter to detect symptomatic AR with surgical indication than other conventional metrics. We retrospectively analyzed patients who underwent elective surgery for severe AR with transesophageal echocardiography (TEE) under anesthesia.

\section{Methods}

\subsection{Patients}

This retrospective study was approved by the institutional review board of our institution; written informed consent was obtained from all participating patients (ERB-C-1144-1). We retrospectively analyzed patients with severe AR who underwent elective aortic valve surgery between June 2015 and December 2018. During the study period, 22 cases were detected. We excluded patients with mitral regurgitation (MR) of moderate or higher grade, coronary syndrome, mental retardation, mild AR, emergent surgery, and adult congenital surgery (Fig. 1). Clinical data including sex, height, body weight, and body surface area, brain natriuretic peptide, and human atrial natriuretic peptide were collected on admission. We also collected patients' echocardiographic data from preoperative transthoracic echocardiography (TTE). Preoperative TTE was performed by the attending cardiologist, and all relevant echo indices were measured in accordance to the American Society of Echocardiography and the Society of cardiovascular anesthesiologist guidelines ${ }^{18,19}$. We divided patients into symptomatic and asymptomatic groups using their admission records. Those of stages above New York Heart Association (NYHA) class I were considered symptomatic and those at NYHA class I stage were asymptomatic. The existence of a symptom was defined according to the medical record as written by nurses and cardiovascular surgeons; these individuals were unaware of the study content.

We also enrolled one patient who was diagnosed with heart failure due to acute severe AR and underwent emergent aortic valve replacement (AVR) to obtain left ventricular vortex information. Written informed consent was obtained from this patient.

\subsection{Echocardiography}

The details of the echocardiogram have been previously reported ${ }^{10}$. After the induction of anesthesia and when the patients' vital signs were stable, TEE was performed by a TEE certified anesthesiologist (AK or KA). We used the Aloka ProSound F75 premier ultrasound machine (Hitachi, Tokyo, Japan). Digitized 2D color Doppler cineloop images obtained in the mid-esophageal left ventricular long axis view by TEE were stored with the VFM configuration before the procedure. These images were transferred to a computer for analysis with VFM software. EL values were averaged over 3 cardiac cycles. When the aliasing phenomenon was detected, the aliasing areas were manually corrected. Measurements of systolic and diastolic EL were calculated as the mean EL during systolic and diastolic phases.

\subsection{Defining Energy Loss}

This technology uses both color Doppler images and speckle tracking images ${ }^{2}$. Intracardiac energy loss can be calculated from the following equation:

Energy Loss $=\int \mu\left\{2\left(\frac{\partial u}{\partial x}\right)^{2}+2\left(\frac{\partial v}{\partial y}\right)^{2}+\left(\frac{\partial u}{\partial y}+\frac{\partial v}{\partial x}\right)^{2}\right\} \mathrm{dA}$

Where $\mu$ is the viscosity of the blood, $u$ and $v$ are velocity components along the Cartesian axes ( $\mathrm{x}$ and $\mathrm{y}$ ), and $\mathrm{A}$ is the area of the unit of the grid. This is a simplified equation that is confined to $2 \mathrm{D}$ flow. An 
example of VFM analysis by TEE is shown (Fig.2). NYHA IV and post AVR cases were not included the main analysis. Figs 3 and 4 show one cardiac EL distribution in NYHA I and NYHA III cases. The main advantage of VFM is that it allows visualization of the turbulent vortex, the area where the blood flow is not laminar with disturbed direction with strong energy dissipation. EL was highest in the region of the left ventricle corresponding to the turbulence produced by the AR jet, as reported previously ${ }^{17}$. The mean EL depends on the left ventricular preload and turbulent left ventricular vortex-like systolic anterior motion ${ }^{20}$. Turbulent flow results in a higher EL than laminar flow, as reported previously ${ }^{21}$.

\subsection{Statistical analysis}

All continuous variables were skewed and summarized by the median and interquartile range. Categorical variables were summarized by frequency and percent. Continuous variables were compared between groups using Mann-Whitney $U$ test. Categorical variables were compared between groups by the Fisher's exact test or chi-square test. Spearman's rank correlation was used to evaluate the correlation between EL and conventional metrics (brain natriuretic peptide, human atrial natriuretic peptide, and major echocardiographic indices). Where data were missing, we excluded them from the analysis (supplementary material, Table S1). All analyses were performed using GraphPad Prism software (Ver.7.00, GraphPad Software, San Diego, CA USA). We defined $\mathrm{p}<0.05$ to indicate statistical significance.

\section{Results}

\subsection{Participants}

During the study period, 15 patients were assessed. There were seven and eight patients in the symptomatic and asymptomatic groups, respectively. There were no statistical differences between the groups' baseline characteristics (Table 1). Details of the diagnosis and symptoms on admission were shown in supplementary material, Table S2.

\subsection{Energy loss and vortex pattern}

The mean EL of one cardiac cycle was higher in the symptomatic (121 [96-184]) than in the asymptomatic group (87 [80-103]) ( $\mathrm{p}=0.040$, Mann-Whitney test), whereas the diastolic diameter was higher in the asymptomatic group (65 [59-78]) than in the symptomatic group (57 [51-57]) ( $\mathrm{p}=0.040$, Mann-Whitney test) (Fig. 5 and supplementary material, Table S3).

We did not detect any statistically significant correlation between the symptomatic and asymptomatic groups in terms of the conventional and other echocardiographic data (Mann-Whitney test, Spearman's rank correlation) (Fig. 6 and supplementary material, Table S3).

\subsection{Energy loss cut off value}

We computed a receiver operating characteristic (ROC) curve for the ability of the mean of one cardiac cycle EL to detect subjective symptoms. Our cutoff of the mean of one cardiac cycle EL value $(95.5 \mathrm{~mW} / \mathrm{m})$ was associated with an increased risk of subjective symptoms in patients with severe AR (Fig. $7 \mathrm{~A}$ ). We also computed a ROC curve for left ventricular end diastolic diameter (LVEDD) (Fig. $7 \mathrm{~B}$ ). LVEDD >58 mm in patients with chronic severe AR rarely complained about subjective symptoms in our cohort.

\section{Discussion}

Our research suggests that left ventricular EL in chronic AR is superior to other conventional echocardiographic indices to explain pathophysiology. The main findings in the current study were as follows: (1) In severe aortic regurgitation, EL may be used to evaluate subjective symptoms more accurately than other conventional metrics; and (2) a left ventricular EL greater than $95.5 \mathrm{~mW} / \mathrm{m}$ in the mid-esophageal long axis view may be a useful diagnostic tool in symptomatic AR.

The novel point is that we investigated the relationship between EL and symptoms in patients with AR who were all in need of surgery and found that a high EL indicated patients' subjective symptoms. There were no significant differences in conventional metrics except for LVEDD, which is a main parameter for surgical 
indication other than subjective symptoms ${ }^{1}$. Since all cases are diagnosed with severe AR, there was no significant difference in preload between the two groups. The reason for EL difference is thought to be vortex interaction between transmitral inflow and AR jet.

Transmitral inflow generates vortex rings which consists of stronger anterior component and weaker posterior components (Fig 8 a) ${ }^{22-25}$. Vortex inside left ventricle, that occurs with transmitral flow known as vortex ring, not only helps smooth mitral closure, but also is believed to support efficient flow ejection toward the outlet with smooth flow turn inside left ventricle ${ }^{22,23}$. According to the previous imaging studies, intraventricular vortex patterns have been known to strongly associated with cardiac performance, and notably, vortex formation caused by transmitral flow plays an important role in diastolic function ${ }^{26-31}$.

By using VFM technology, vortex changes generated by the collision of AR jet and intramitral blood flow can be visualized. Morisawa et al, reported paravalvular leakage (PVL) jet of aortic valve affects intraventricular vortex formation, and the jet direction also affects intraventricular vortex formation and vortex interaction between the transmitral flow and PVL jet ${ }^{26}$. When the AR jet collided with the transmitral flow and merging, the patient would be less symptomatic (Fig 8 b, d). In symptomatic patients, the AR jet would interfere the consistence of left ventricular vortex ring, causing higher EL than that noted in asymptomatic condition (Fig $8 \mathrm{c}$ ). A more detailed investigation about the disturbance of left ventricular vortex by AR jet and its association with EL elevation warrant further study.

Our research finding that asymptomatic patients' diastolic diameter was higher than that in symptomatic patients reflects the fact that many asymptomatic patients underwent surgery for AR because of left ventricular enlargement. Our ROC analysis revealed that the larger the left ventricle, the less the patient complains of symptoms, if properly treated (Fig.7 B). Because they had no symptoms, the objective indications for surgery were limited; in the asymptomatic patient, progressive left ventricular dilatation and adaptation results from AR volume overload. Finally, such patients have poor cardiac function and poor surgical outcomes ${ }^{32}$.

If we were to continue to monitor patients by using left ventricular EL, it would be easier to define the indications for surgery. Therefore, we defined a threshold value in patients with symptomatic AR. This is higher than the normal patient reference value ${ }^{6}$. When inefficient, unphysiological flow occurs in the left ventricle, the EL rises and the heart tries to compensate. However, if the heart fails to compensate, heart failure ensues; if severe, EL may decrease owing to insufficient left ventricular energy generation ${ }^{4}$. We, therefore, consider it necessary to evaluate patients' EL continuously.

Qualification of patient workload can be a good tool for treatment decision making. More large, prospective, randomized studies are needed to clarify the relationship between the progression of disease and EL. In the future, we should identify whether the AR jet causes heart failure and whether the patient has a surgical indication based on the intracardiac blood flow dynamics and EL change. The authors believe that this research demonstrates the therapeutic impact of monitoring left ventricular EL in AR.

There are several limitations to this study. First, our study is limited in that it was a retrospective, nonrandomized study assessing a relatively small cohort. It takes time to investigate whether EL really has a useful role in prognosis prediction. Further studies assessing a large population cohort are required to validate our findings. Second, as we primarily analyzed two-dimensional images, with software that assumed 2D flow, actual regurgitation jet is complex $3 \mathrm{D}$ flow, and a three-dimensional evaluation is needed to ensure accurate assessment of the severity of $\mathrm{AR}^{13,17}$. Three-dimensional VFM is currently unavailable. A more advanced software that can overcome this limitation needs to be developed. Third, these data were analyzed by TEE and were from patients who were under general anesthesia. TEE provide superior visualization of the left ventricle without the intervening lung and bone compared with TTE. There is modest agreement in AR assessment between TTE and intraoperative $\mathrm{TEE}^{33}$. However, there is limited information on the difference between TEE and TTE in terms of left ventricular EL analysis. Since TTE is also important in clinical practice, it is necessary to analyze TTE data in the future. Fourth, all patients in this research were already considered suitable to undergo surgery; we should also analyze patients who are not. 


\section{Conclusion}

In summary, EL calculated by VFM can quantify patients' subjective symptoms more accurately than other conventional metrics in chronic AR. VFM can visualize the interference of the AR jet with intra-mitral flow and alteration of the intraventricular vortex direction, which increase the cardiac workload. Large prospective studies are necessary to further assess the utility of this technique for patients with AR.

\section{References}

1. Nishimura RA, Otto CM, Bonow RO, Carabello BA, Erwin JP 3rd, Guyton RA, et al. 2014 AHA/ACC Guideline for the Management of Patients With Valvular Heart Disease: executive summary: a report of the American College of Cardiology/American Heart Association Task Force on Practice Guidelines. Circulation. 2014;129:2440-2492.

2. Itatani K, Okada T, Uejima T, Tanaka T, Ono M, Miyaji K, et al. Intraventricular flow velocity vector visualization based on the continuity equation and measurements of vorticity and wall shear stress. Jpn J Appl Phys.

3. Garcia D, del Alamo JC, Tanne D, Yotti R, Cortina C, Bertrand E, et al. Two-dimensional intraventricular flow mapping by digital processing conventional color-doppler echocardiography images. IEEE Trans Med Imaging. 2010;29:1701-1713.

4. Itatani K. When the blood flow becomes bright. Eur Heart J. 2014;35:747-752a.

5. Hayashi T, Itatani K, Inuzuka R, Shimizu N, Shindo T, Hirata Y, et al. Dissipative energy loss within the left ventricle detected by vector flow mapping in children: Normal values and effects of age and heart rate. J Cardiol. 2015;66:403-410.

6. Akiyama K, Maeda S, Matsuyama T, Kainuma A, Ishii M, Naito Y, et al. Vector flow mapping analysis of left ventricular energetic performance in healthy adult volunteers. BMC Cardiovasc Disord. 2017;17:21.

7. Mori S, Isotani A, Yamaji K, Yano M, Morinaga T, Hayashi M, et al. Device Thrombosis After Percutaneous Edge-to-Edge Mitral Valve Repair. JACC Cardiovasc Interv. 2020;13:653-654.

8. Rodriguez Muñoz D, Moya Mur JL, Becker Filho DC, Rincon Díaz LM, Gonzalez Gómez A, FernándezGolfín C, et al. Flow Mapping Inside a Left Ventricular Aneurism: A Potential Tool to Demonstrate Thrombogenicity. Echocardiography. 2013;31:E10-E12.

9. Nakashima K, Itatani K, Kitamura T, Oka N, Horai T, Miyazaki S, et al. Energy dynamics of the intraventricular vortex after mitral valve surgery. Heart Vessels. 2017;32:1123-1129.

10. Akiyama K, Nakamura N, Itatani K, Naito Y, Kinoshita M, Shimizu M, et al. Flow-dynamics assessment of mitral-valve surgery by intraoperative vector flow mapping. Interact Cardiovasc Thorac Surg. 2017;24:869875.

11. Yoshida S, Miyagawa S, Fukushima S, Yoshikawa Y, Hata H, Saito S, et al. Cardiac function and type of mitral valve surgery affect postoperative blood flow pattern in the left ventricle. Circ J. 2018;83:130-138.

12. Hayashi H, Akiyama K, Itatani K, DeRoo S, Sanchez J, Ferrari G, et al. A novel in vivo assessment of fluid dynamics on aortic valve leaflet using epi-aortic echocardiogram. Echocardiography. 2020;37:323-330.

13. Honda T, Itatani K, Takanashi M, Kitagawa A, Ando H, Kimura S, et al. Exploring energy loss by vector flow mapping in children with ventricular septal defect: Pathophysiologic significance. Int J Cardiol. $2017 ; 244: 143-150$.

14. Honda T, Itatani K, Miyaji K, Ishii M. Assessment of the vortex flow in the post-stenotic dilatation above the pulmonary valve stenosis in an infant using echocardiography vector flow mapping. Eur Heart J. 2013;35:306. 
15. Kainuma A, Akiyama K, Naito Y, Hayase K, Hongu H, Itatani K, et al. Energetic performance index improvement after Glenn and Damus-Kaye-Stansel procedure using vector flow mapping analysis: a case report. JA Clin Rep. 2020;6:5.

16. Mele D, Smarrazzo V, Pedrizzetti G, Capasso F, Pepe M, Severino S, et al. Intracardiac Flow Analysis: Techniques and Potential Clinical Applications. J Am Soc Echocardiogr. 2019;32:319-332.

17. Stugaard M, Koriyama H, Katsuki K, Masuda K, Asanuma T, TakedaY, et al. Energy loss in the left ventricle obtained by vector flow mapping as a new quantitative measure of severity of aortic regurgitation: a combined experimental and clinical study. Eur Heart J Cardiovasc Imaging. 2015;16:723-730.

18. Zoghbi WA, Enriquez-Sarano M, Foster E, Grayburn PA, Kraft CD, Levine RA, et al. Recommendations for evaluation of the severity of native valvular regurgitation with two-dimensional and doppler echocardiography. J Am Soc Echocardiogr. 2003;16:777-802.

19. Hahn RT, Abraham T, Adams MS, Bruce CJ, Glas KE, Lang RM, et al. Guidelines for Performing a Comprehensive Transesophageal Echocardiographic Examination: Recommendations from the American Society of Echocardiography and the Society of Cardiovascular Anesthesiologists. J Am Soc Echocardiogr. 2013;26:921-964.

20. Akiyama K, Naito Y, Kinoshita M, Ishii M, Nakajima Y, Itatani K, et al. Flow Energy Loss Evaluation in a Systolic Anterior Motion Case After the Ross Procedure. J Cardiothorac Vasc Anesth.2017;31:2118-2122.

21. Shibata M, Itatani K, Hayashi T, Honda T, Kitagawa A, Miyaji K, et al. Flow energy loss as a predictive parameter for right ventricular deterioration caused by pulmonary regurgitation after tetralogy of Fallot repair. Pediatr Cardiol. 2018;39:731-742.

22. Kilner PJ, Yang GZ, Wilkes AJ, Mohiaddin RH, Firmin DN, Yacoub MH. Asymmetric redirection of flow through the heart. Nature. 2000;404:759-761. doi:10.1038/35008075

23. Pedrizzetti G, Domenichini F. Nature Optimizes the Swirling Flow in the Human Left Ventricle. Phys Rev Lett. 2005;95:108101.

24. Domenichini F, Querzoli G, Cenedese A, Pedrizzetti G. Combined experimental and numerical analysis of the flow structure into the left ventricle. J Biomech. 2007;40:1988-1994.

25. Rodriguez Munoz D, Markl M, Moya Mur JL, Barker A, Fernandez-Golfin C, Lancellotti P, et al. Intracardiac flow visualization: current status and future directions. Eur Heart J Cardiovasc Imaging. 2013;14:1029-1038.

26. Morisawa D, Falahatpisheh A, Avenatti E, Little SH, Kheradvar A. Intraventricular Vortex Interaction between Transmitral Flow and Paravalvular Leak. Sci Rep. 2018;8:15657.

27. Kheradvar A, Houle H, Pedrizzetti G, Tonti G, Belcik T, Ashraf M, et al. Echocardiographic Particle Image Velocimetry: A Novel Technique for Quantification of Left Ventricular Blood Vorticity Pattern. J Am Soc Echocardiogr. 2010;23:86-94.

28. Kheradvar A, Assadi R, Falahatpisheh A, Sengupta PP. Assessment of Transmitral Vortex Formation in Patients with Diastolic Dysfunction. J Am Soc Echocardiogr. 2012;25:220-227.

29. Abe H, Caracciolo G, Kheradvar A, Pedrizzetti G, Khandheria BK, Narula J, et al. Contrast echocardiography for assessing left ventricular vortex strength in heart failure: a prospective cohort study. Eur Heart J Cardiovasc Imaging. 2013;14:1049-1060.

30. Hong GR, Pedrizzetti G, Tonti G, Li P, Wei Z, Kim JKet al. Characterization and Quantification of Vortex Flow in the Human Left Ventricle by Contrast Echocardiography Using Vector Particle Image Velocimetry. JACC Cardiovasc Imaging. 2008;1:705-717. 
31. Falahatpisheh A, Pahlevan NM, Kheradvar A. Effect of the Mitral Valve's Anterior Leaflet on Axisymmetry of Transmitral Vortex Ring. Ann Biomed Eng. 2015;43:2349-2360.

32. Chaliki HP, Mohty D, Avierinos JF, Scott CG, Schaff HV, Tajik AJ, et al. Outcomes After Aortic Valve Replacement in Patients With Severe Aortic Regurgitation and Markedly Reduced Left Ventricular Function. Circulation. 2002;106:2687-2693.

33. Neuman YM, Brasch AV, Kobal S, Khan SS, Mirocha JM, Naqvi TZ, et al. Comparison of Transthoracic and Intraoperative Transesophageal Color Flow Doppler Assessment of Mitral and Aortic Regurgitation. Cardiology. 2003;99:145-152.

Fig 1. Study flow chart.

* We enrolled patients who was diagnosed heart failure caused by acute severe AR and underwent emergent surgery to obtain left ventricular vortex information.

Fig 2. Visualization of blood flow by vector flow mapping.

Intraventricular vector flow mapping in the patient at the stages NYHA I (Asymptomatic), II-IV (Symptomatic), and post AVR. E) Early diastole phase. L) Late diastole phase. Abbreviations: Ao, Aorta, LA, left atrium.

Fig 3. Visualization of blood flow energy loss and blood flow by vector flow mapping.

These figures show EL. Brightness indicates high energy loss. The AR jet collides with mitral inflow, which causes left ventricular vortex turbulence and high dissipative EL. A) Asymptomatic case (NYHA I), B) Symptomatic case (NYHA III). Abbreviations: Early diastole, The early phase of the diastolic period; Systolic, Systolic phase; IVCT, The isovolumic contraction phase; IVRT, The isovolumic relaxation phase; Late diastole, The late phase of the diastolic period.

Fig 4. The graph shows an example of intraventricular flow energy loss distribution during one cardiac cycle.

The blue curve represents the asymptomatic case (NYHA I) and red represents the symptomatic case (NYHA III). Abbreviations: MC, mitral valve closing; MO, mitral valve opening.

Fig 5. Main findings of this study.

Black dots represent symptomatic patients, whereas white dots represent asymptomatic patients. The mean energy loss of one cardiac cycle is higher in the symptomatic group than in the asymptomatic group ( $\mathrm{p}=0.040)$ (A). The diastolic diameter is higher in the asymptomatic group than in the symptomatic group $(\mathrm{p}=0.040)$ (B). mEL: mean energy loss in one cardiac cycle.

Fig 6. Correlations between mean energy loss in one cardiac cycle and other parameters.

There are no statistical correlations between the mean energy loss in one cardiac cycle and other parameters. a) $\mathrm{mEL}$ and brain natriuretic peptide (BNP). b) mEL and human atrial natriuretic peptide (hANP). c) mEL and left ventricular ejection fraction (LVEF). d) mEL and LVEDD. e) mEL and left ventricular end-systolic diameter (LVESD). f) mEL and regurgitation volume (RV). g) mEL and pressure half time (PHT). h) $\mathrm{mEL}$ and effective regurgitant orifice (ERO). i) mEL and Vena Contracta (VC).

Fig $7 \mathrm{~A}$ and B. Receiver operating characteristic curve for revealing association between mEL, LVEDD, and patient subjective symptoms.

Our cutoff value $(\mathrm{mEL}=95.5 \mathrm{~mW} / \mathrm{m}, \mathrm{LVEDD}=58 \mathrm{~mm})$ is that value which corresponds to a point on the receiver operating characteristic curve nearest to the upper corner of the graph. Abbreviations: AUC, area under curve.

Fig 8. Schematics of LV vortex interaction in diastole. 
a) Intramitral flow organized itself in a circulatory pattern that redirects the flow to the aorta. b) The AR jet collided with the transmitral flow and merging. c)The AR jet inhibits the formation of optimal left ventricular vortex. d) The LV cavity is enlarged, and AR jet and transmitral flow are merging. Abbreviations: LA, left atrium; Ao, aorta; LV, left ventricle.

Table 1. Patients' characteristics

\begin{tabular}{llll}
\hline & $\begin{array}{l}\text { Asymptomatic group } \\
(\mathrm{n}=8)\end{array}$ & $\begin{array}{l}\text { Symptomatic group } \\
(\mathrm{n}=7)\end{array}$ & $P$ value \\
\hline Age (years) & $48[39$ to 74$]$ & $65[54$ to 68$]$ & 0.694 \\
Sex (female) & $1(12.5)$ & $2(28.5)$ & 0.569 \\
Height $(\mathrm{cm})$ & $174[169$ to 180$]$ & $165[158$ to 172$]$ & 0.198 \\
Weight $(\mathrm{kg})$ & $70[65$ to 78$]$ & $62[51$ to 70$]$ & 0.219 \\
Body surface area $\left(\mathrm{m}^{2}\right)$ & $1.9[1.8$ to 2.0$]$ & $1.7[1.5$ to 1.9$]$ & 0.179 \\
Past medical history & & & \\
Hypertension & $1(12.5)$ & $4(57.1)$ & 0.067 \\
Chronic kidney disease & $0(0)$ & $1(14.3)$ & 0.269 \\
$\begin{array}{l}\text { Dyslipidemia } \\
\text { Paroxysmal atrial }\end{array}$ & $0(0)$ & $1(14.3)$ & 0.269 \\
fibrillation & $1(12.5)$ & $0(0)$ & 0.333 \\
Post heart failure & $4(50)$ & $1(14.3)$ & 0.282 \\
$\begin{array}{l}\text { Bicuspid valve } \\
\text { NYHA class }\end{array}$ & $4(50)$ & $1(14.3)$ & 0.282 \\
I & $8(100)$ & $0(0)$ & $<0.001^{*}$ \\
II & $0(0)$ & $6(85.7)$ & \\
III & $0(0)$ & $1(14.3)$ & \\
$\begin{array}{l}\text { Other valvular disease } \\
\text { mild PR }\end{array}$ & $0(0)$ & $1(14.3)$ & \\
mild TR, mild PR & $1(12.5)$ & $1(14.3)$ & \\
mild TR, mild MR & $1(12.5)$ & $4(57.1)$ & \\
mild PR, mild MR & $1(12.5)$ & $0(0)$ & \\
mild TR, mild PR, & $3(37.5)$ & $1(14.3)$ & \\
mild MR & & & \\
\hline
\end{tabular}

Baseline characteristics of patients. Data are presented as n (\%) or median [IQR]. The characteristics of patients did not show any significant intergroup difference. Abbreviations: MR, mitral regurgitation; NYHA, New York Heart Association; PR, pulmonary regurgitation; TR, tricuspid regurgitation. 


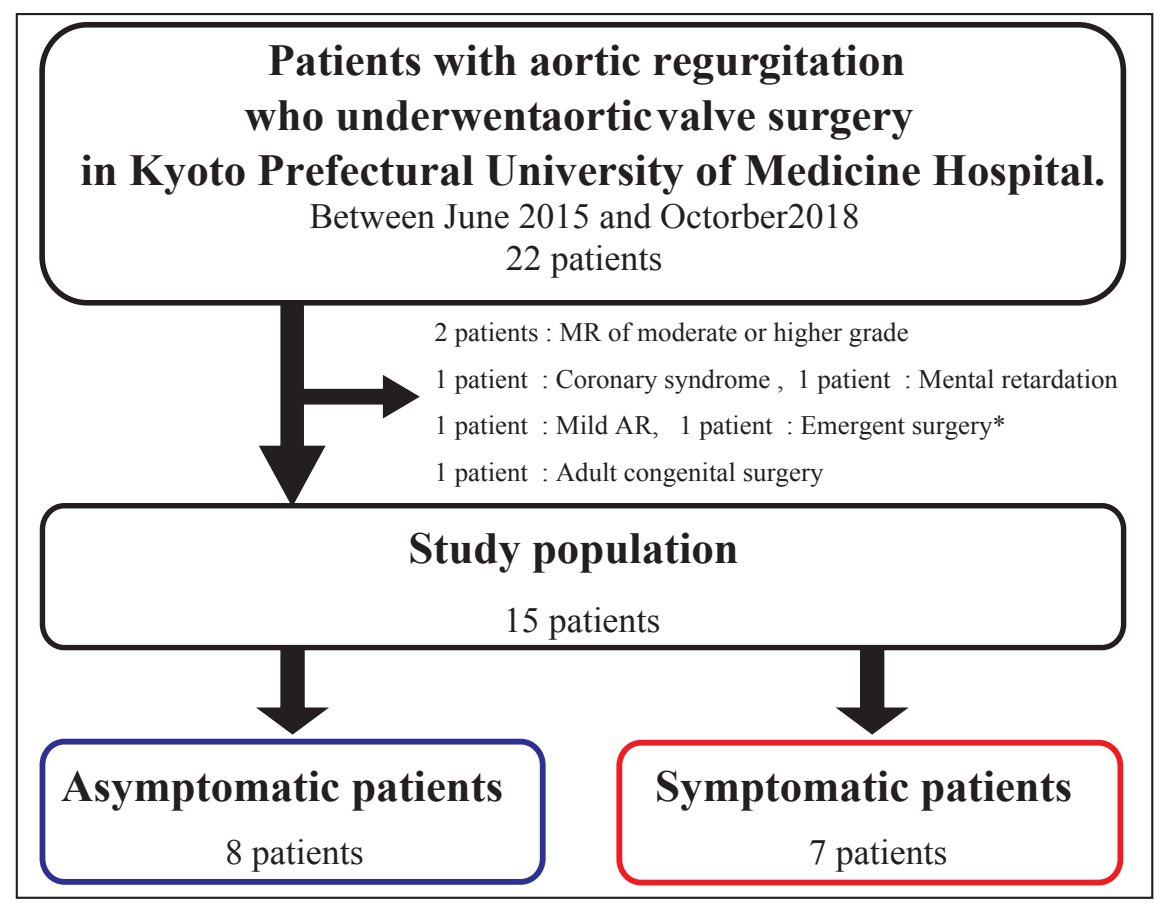

\section{Hosted file}

Fig 2 AR.pdf available at https://authorea.com/users/352647/articles/476804-preoperativeleft-ventricular-energy-loss-in-the-operating-theater-reflects-subjective-symptoms-inchronic-aortic-regurgitation

Hosted file

Figure 3.pdf available at https://authorea.com/users/352647/articles/476804-preoperativeleft-ventricular-energy-loss-in-the-operating-theater-reflects-subjective-symptoms-inchronic-aortic-regurgitation

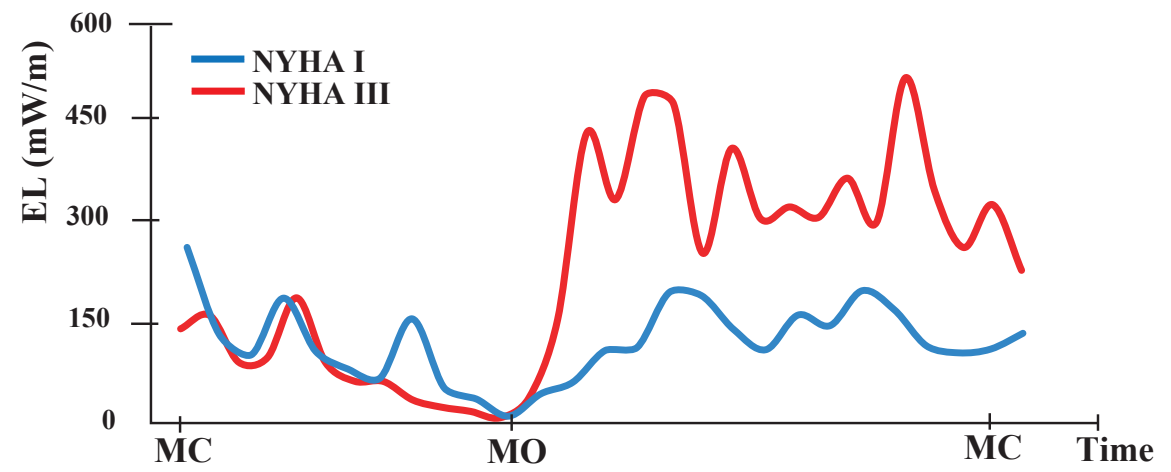



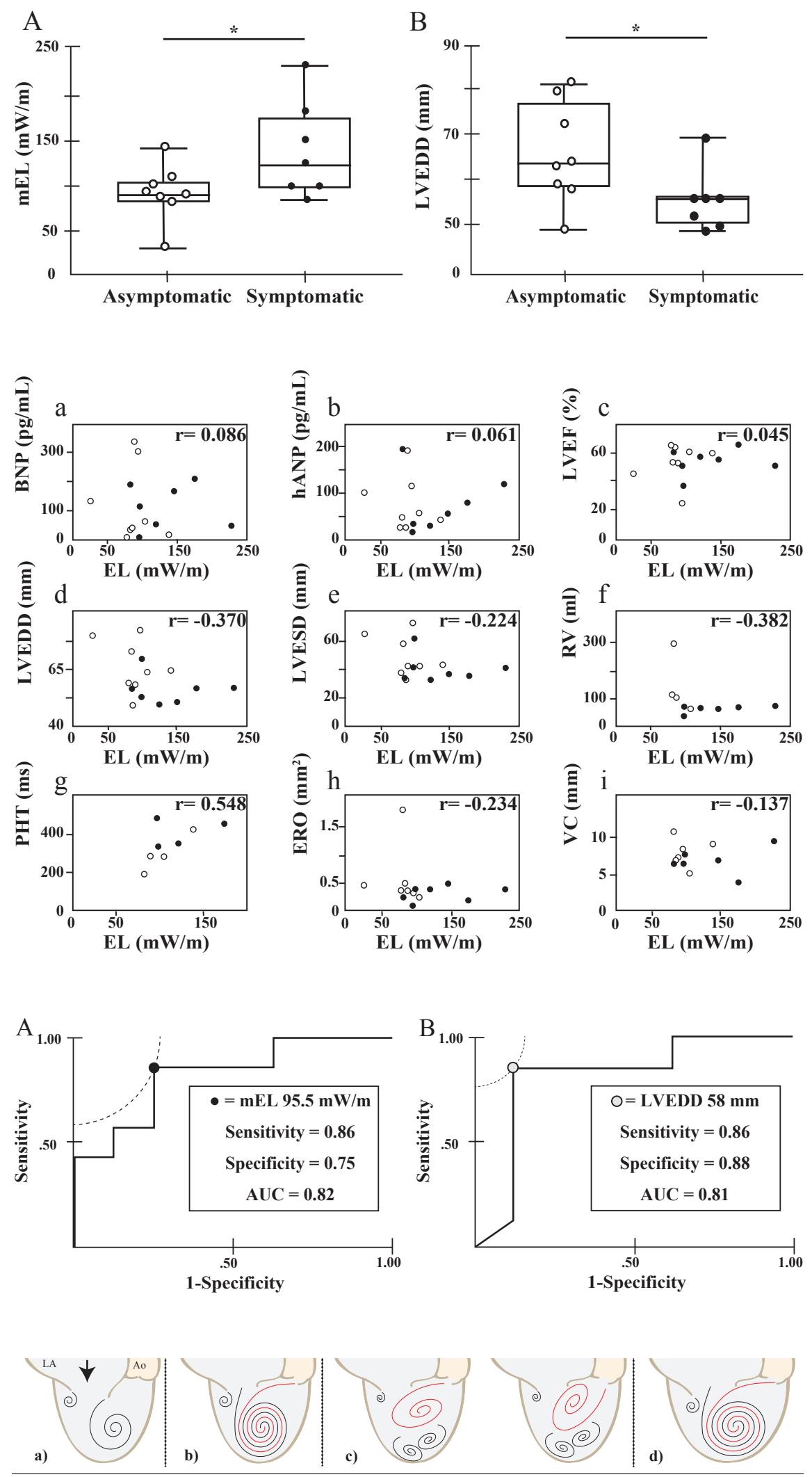


\section{Hosted file}

Table 1 EL AR.docx available at https://authorea.com/users/352647/articles/476804preoperative-left-ventricular-energy-loss-in-the-operating-theater-reflects-subjectivesymptoms-in-chronic-aortic-regurgitation 